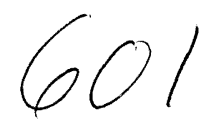

\title{
Intraoperative Changes in Blood Coagulation and Thrombelastographic Monitoring in Liver Transplantation
}

\author{
Yoo Goo Kang, MD, Douglas J. Martin, MD, Jose Marquez, MD, Jessica H. Lewis, MD, \\ Franklin A. Bontempo, MD, Byers W. Shaw Jr, MD, Thomas E. Starzl, MD, and
}

Peter M. Winter, MD

\begin{abstract}
KANG YG, MARTIN DJ, MARQUEZ J, LEWIS JH, BONTEMPO FA, SHAW BW Jr, STARZL TE, WINTER PM. Intraoperative changes in blood coagulation and thrombelastographic monitoring in liver transplantation. Anesth Analg 1985;64:888-96.

The blood coagulation system of 66 consecutive patients undergoing consecutive liver transplantations was monitored by thrombelastograph and analytic coagulation profile. A poor preoperative coagulation state, decrease in levels of coagulation factors, progressive fibrinolysis, and whole blood clot lysis were observed during the preanhepatic and anhepatic stages of surgery. A further general decrease in coagulation factors and platelets, activation of fibrinolysis,
\end{abstract}

and abrupt decrease in levels of factors $V$ and VIII occurred before and with reperfusion of the homograft. Recovery of blood coagulability began 30-60 min after reperfusion of the graft liver, and coagulability had returned toward baseline values $2 \mathrm{hr}$ after reperfusion. A positive correlation was shown between the variables of thrombelastography and those of the coagulation profile. Thrombelastography was shown to be a reliable and rapid monitoring system. Its use was associated with a $33 \%$ reduction of blood and fluid infusion volume, whereas blood coagulability was maintained without an increase in the number of blood product donors.

Key Words: BLOOD-coagulation. LIVER_transplantation.
Liver transplantation, which began as highly experimental surgery $20 \mathrm{yr}$ ago, is now recognized as a major means of therapy for patients with end-stage liver disease (1). However, massive blood loss during liver transplantation is still a major concern. The liver produces most of the blood coagulation factors, so it is not surprising that we see very low levels of these factors and prolonged prothrombin time (PT) and activated partial thromboplastin time (aPTT) in many patients receiving liver transplants (2). Frequently we have seen thrombocytopenia, which may result from gastrointestinal bleeding, splenomegaly, or malnutrition (3). At the same time, numerous collateral channels and portal hypertension, together with increased capillary fragility, make maintenance of surgical hemostasis very difficult.

Previous studies on the blood coagulation system in humans and in animals undergoing liver trans-

Received from the Departments of Anesthesiology, Medicine, and Surgery, University of Pittsburgh School of Medicine and the Central Blood Bank of Pittsburgh, Pittsburgh, Pennsylvania. Accepted for publication April 4, 1985.

Address correspondence to Dr. Kang, Department of Anesthesiology, Presbyterian University Hospital, De Soto and O'Hara Street, Pittsburgh, PA 15213. plantation have demonstrated dilutional coagulopathy associated with massive blood transfusion, decreased fibrinogen levels, and thrombocytopenia $(4,5)$. Fibrinolysis, beginning during the anhepatic stage of surgery and becoming "explosive" on reperfusion of the homograft, has been reported (6). A consumption coagulopathy accompanied by an increased level of fibrin degradation products appeared to play a major role (7). However, these observations were limited to a few patients in the early era of liver transplantation, and comprehensive information is still lacking.

Another difficulty in the intraoperative management of liver transplantation has been actively monitoring the coagulation system and determining the appropriate treatment during dynamic blood volume changes. The use of the thrombelastograph (TEG) was suggested by von Kaulla et al. (4) and Howland et al. (8). However, the efficacy of thrombelastographic monitoring of blood coagulation during liver transplantation has not been established.

The purpose of our study was to investigate the changes in the blood coagulation system, the clinical applicability of thrombelastography as a monitoring system, and the clinical effectiveness of thrombelastography-guided replacement therapy in a large group of patients undergoing liver transplantation. 


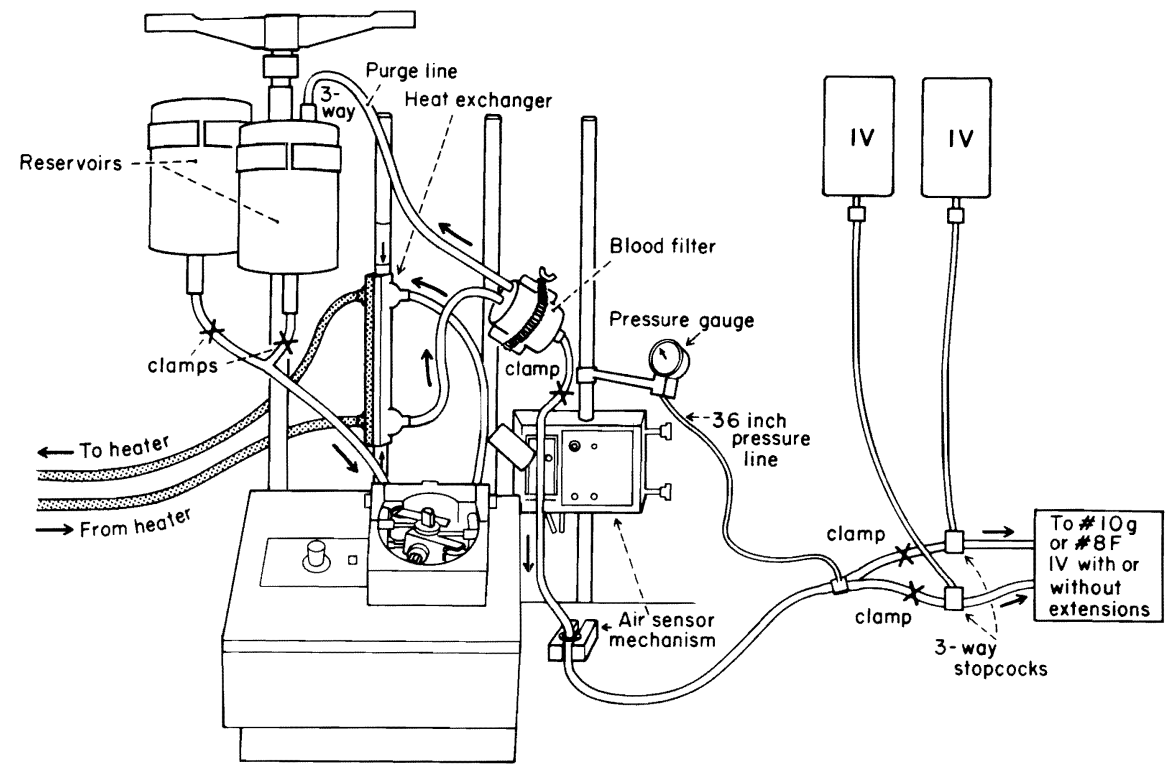

Figure 1. A rapid infusion system, developed by JJ Sassano, MD, of the University of Pittsburgh School of Medicine.

\section{Methods}

Patients undergoing liver transplantation between February 1983 and January 1984 were observed as prospective members for the study. All patients had two indwelling 8.5-French catheters inserted for volume infusion, one in an antecubital vein and another in an internal or an external jugular vein. In addition, a flow-directed pulmonary arterial catheter was inserted via the right internal jugular vein, and an indwelling catheter was placed in a radial artery. Normal saline solution without heparin was used for continuous flushing of the pressure-monitoring catheters to avoid anticoagulant effects. A rapid infusion system was developed (by JJ Sassano, University of Pittsburgh School of Medicine), which was able to deliver warmed, premixed fluid at a rate of up to 2000 $\mathrm{ml} / \mathrm{min}$ (Fig. 1). This device was used to replace lost blood volume with a fluid composed of red blood cells $(\mathrm{RBC})$, fresh frozen plasma (FFP), and electrolyte solution (Plasma-lyte A, Travenol Laboratories Inc., Deerfield, IL 60015) in a ratio of 300:200:250 ml. All blood products were preserved with citrate phosphate and dextrose with adenine (CPDA-1). Intraoperative volume infusion was guided by systolic blood pressure, heart rate, and pulmonary capillary occlusion pressure measurements. Arterial blood gas tensions and serum electrolytes, glucose, and ionized calcium levels were monitored every hour, though more frequently if clinically indicated.

Coagulation tests employed previously described methods (9-11) and were performed for patient care purposes at times when sudden or profound changes were anticipated. Blood samples $(15 \mathrm{ml}$ each) were obtained at various intervals: before induction of anesthesia; $30 \mathrm{~min}$ after beginning the operation; thereafter every $2 \mathrm{hr}$ or after infusion of every $6 \mathrm{~L}$ of blood volume; $5 \mathrm{~min}$ into the anhepatic stage; $30 \mathrm{~min}$ into the anhepatic stage; $5 \mathrm{~min}$ after reperfusion of the homograft; $30 \mathrm{~min}$ after reperfusion; and thereafter every $2 \mathrm{hr}$ or after infusion of every $6 \mathrm{~L}$ of blood volume. The blood coagulation profile included measurements of PT; aPTT; thrombin time (TT); reptilase time; levels of factors I, II, V, VII, VIII, IX, X, XI, and XII; fibrin degradation product (FDP) level; euglobulin lysis time (ELT); and platelet count.

At the same time, whole blood was used for thrombelastographic monitoring of blood coagulation (12). Whole blood $(0.36 \mathrm{ml})$ was placed in the Thrombelastograph D (Hellige Company, Freiburg, Germany), and four drops of mineral oil were spread over the blood surface to prevent evaporation of blood. The recording began $4 \mathrm{~min}$ from the blood sampling time. The TEG is shown schematically in Figure 2. It repeatedly measures the shear elasticity of a blood clot from the time when the first fibrin strands are formed to the completion of the clot formation, including fibrinolysis. An oscillating cup contains $0.36 \mathrm{ml}$ of whole blood kept at $37^{\circ} \mathrm{C}$. A pin, suspended by a torsion wire, is lowered into the blood specimen in the cup. While the blood remains fluid, the container motion does not influence the pin. Once the clot begins to form, the fibrin strands gradually strengthen their hold on the cup and pin. Therefore, the cup is coupled to the pin; and the shear elasticity of the blood clot, which is transmitted to the pin, is recorded on thermal paper. The TEG records the activity of whole blood coagulation (13), including cellular and humoral ele- 


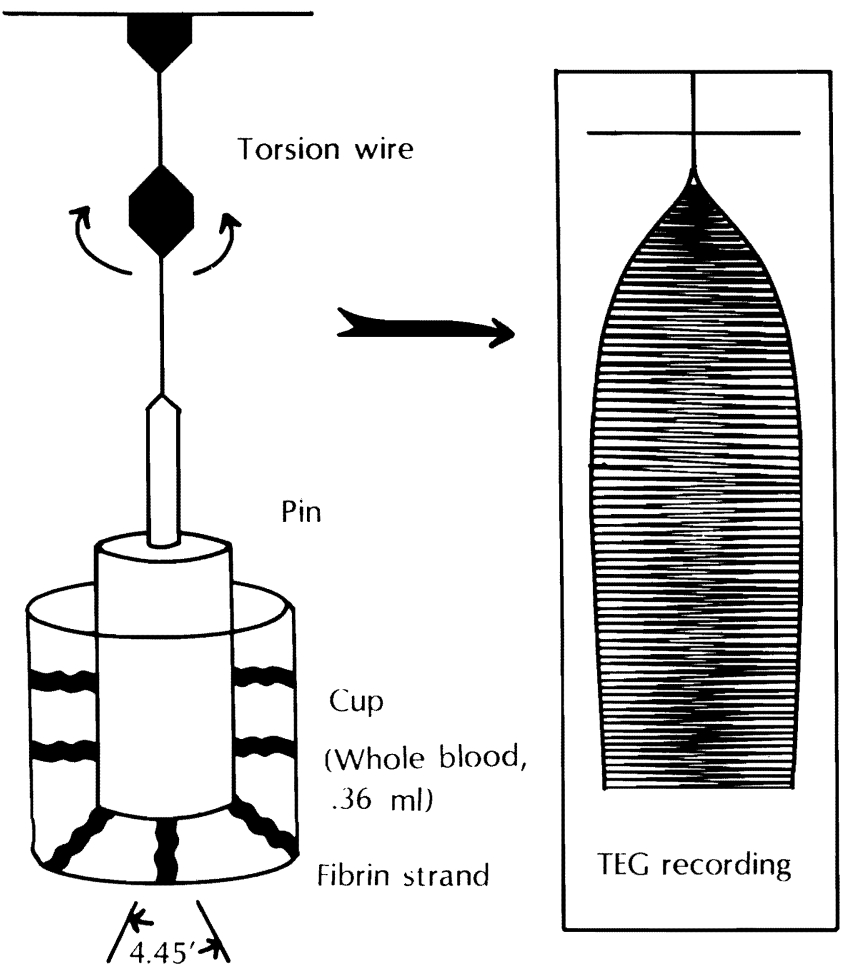

Figure 2. Thrombelastography: sensitive bedside monitoring of coagulation. It measures the shear elasticity of a blood clot from the time when the first fibrin strands are formed to the completion of the clot formation, including fibrinolysis.

ments such as interactions of $\mathrm{RBC}$, platelets, coagulation factors, and calcium. The following variables were measured (Fig. 3): reaction time $(R, \min )$; maximum amplitude $(M A, \mathrm{~mm})$; amplitude $60 \mathrm{~min}$ after $M A\left(A_{60}, \mathrm{~mm}\right)$; coagulation time $(R+k, \mathrm{~min})$; clot formation rate $\left(\alpha,{ }^{\circ}\right)$; blood clot lysis time $(F, \min )$; and blood clot lysis index $\left(A_{60} / M A \times 100, \%\right)$.

The blood product replacement therapy was guided by the results of thrombelastographic monitoring. A prolonged reaction time $(R>15 \mathrm{~min})$ was treated with 2 units of FFP and a decrease in maximum amplitude $(M A<40 \mathrm{~mm})$, with 10 units of platelets. Cryoprecipitate (6 units) was infused when the above therapy did not improve coagulation or when the clot formation rate was slow $\left(\alpha<45^{\circ}\right)$. However, because the anhepatic stage of surgery included veno-venous bypass (femoral and portal vein to axillary vein) without systemic heparinization, infusion of platelets and cyroprecipitate was witheld during that stage to lessen the chance of thromboembolism. To evaluate the clinical effectiveness of TEG-guided replacement therapy, the blood volume infused in these patients (TEGmonitored group) was compared with that of a group of patients who received liver transplants between
August 1981 and January 1983 without TEG monitoring (nonmonitored group).

The measurements of all the coagulation factor levels, thrombelastographic variables, and infused blood product volumes were analyzed by analysis of variance of repeated measures, nonparametric Wilcoxon test, linear regression, and $\chi^{2}$-analysis. $P<0.05$ was considered statistically significant.

\section{Results}

Sixty-six adult patients received liver transplants between February 1983 and January 1984. Three patients for whom data were insufficient were excluded from the analysis. The surgical procedure was arbitrarily divided into three distinct stages: stage I, isolation of the host liver; stage II, the anhepatic stage; and stage III, postreperfusion of the graft liver.

\section{Changes in Coagulation Profile}

Patients were divided into six groups according to the etiology of their underlying disease: primary biliary cirrhosis, postnecrotic cirrhosis, sclerosing cholangitis, neoplasm, miscellaneous liver diseases, and retransplantation. Preoperative diagnoses in the miscellaneous group were cryptogenic cirrhosis, acute chemical hepatitis, Wilson's disease, hemochromatosis, and $\alpha 1$-antitrypsin deficiency. Preoperative coagulation profiles were abnormal in a large proportion of patients: PT in $41(65 \%)$, aPTT in $45(71 \%)$, factor I level in $9(14 \%)$, factor $V$ level in $37(59 \%)$, factor VII level in $35(56 \%)$, factor VIII level in $2(3 \%)$, and platelet count in $44(70 \%)$. ELT was less than $2 \mathrm{hr}$ in 17 patients $(27 \%)$, and FDP was positive in 15 patients $(24 \%)$. The mean preoperative coagulation profile of each disease group is shown in Table 1. PT was prolonged in patients of all groups, and it was remarkably prolonged in patients in the postnecrotic cirrhosis and miscellaneous disease groups. aPTT was prolonged in all patients except those with neoplasms. The platelet count was significantly decreased in patients with sclerosing cholangitis and in the miscellaneous disease group. All patients except those in the miscellaneous group demonstrated a relatively normal fibrinogen level. The level of factor $\mathrm{V}$ was higher than $0.5 \mathrm{U} / \mathrm{ml}$ in the patients of the neoplasm, primary biliary cirrhosis, and retransplantation groups. Factor VIII level was higher than $1.0 \mathrm{U} / \mathrm{ml}$ in all but six patients.

Intraoperative coagulation profile and thrombelastographic measurements of patients with primary biliary cirrhosis ( 11 patients) are shown in Table 2. During stage I of surgery, PT and aPTT remained prolonged. All the coagulation factor levels, including 


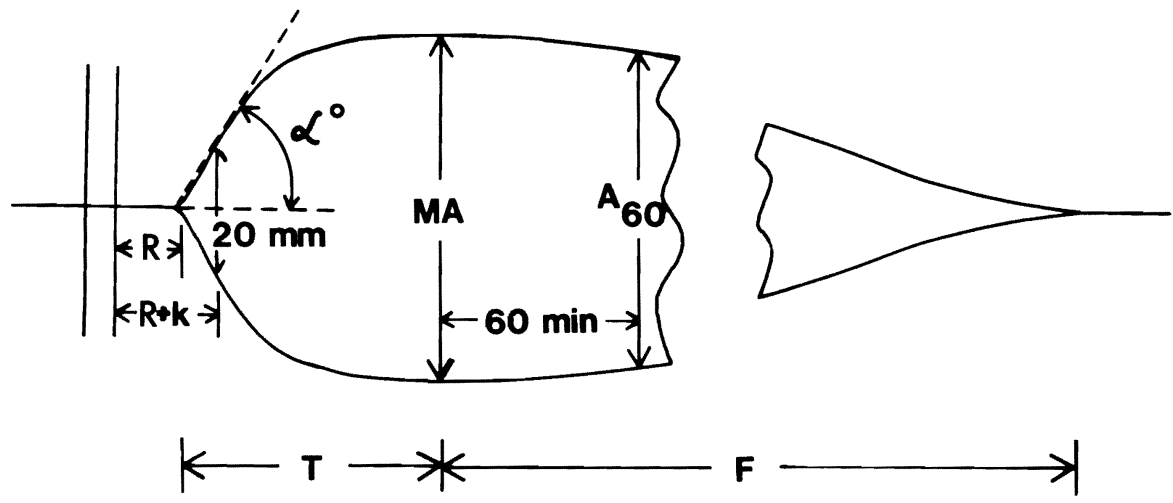

Figure 3. Variables and normal values measured by thrombelastography. Abbreviations: $R$-reaction time, $6-8 \mathrm{~min} ; R+$ $k$-coagulation time, $10-12 \mathrm{~min} ; \alpha$-clot formation rate, $>50^{\circ} ; M A$-maximum amplitude, $50-70 \mathrm{~mm} ; A_{60}$-amplitude 60 min after $M A ; A_{60} / M A \cdot 100$-whole blood clot lysis index, $>85 \%$; and $F$-whole blood clot lysis time, $>300 \mathrm{~min}$.

the platelet count, decreased progressively during surgery. In stage II (anhepatic stage) the levels of all coagulation factors did not change significantly, although aPTT was progressively prolonged and ELT became shortened. On reperfusion of the homograft (stage III $+5 \mathrm{~min}$ ), PT and aPTT were remarkably prolonged, and levels of all other coagulation factors were significantly decreased. With the progress of surgery, coagulation profiles improved slowly, beginning $30 \mathrm{~min}$ after reperfusion (III $+30 \mathrm{~min}$ ). At the end of surgery, PT and aPTT returned toward preoperative values, and platelet counts and the levels of coagulation factors, except factor $\mathrm{V}$, returned toward clinically acceptable levels. The level of factor $\mathrm{V}, 0.23 \pm 0.13 \mathrm{U} / \mathrm{ml}$, was the lowest of the coagulation factors at the conclusion of surgery. Reptilase time and TT were measured in all 11 patients; both were moderately prolonged in all these patients, indicating a possible dysfibrinogenemia. A remarkably prolonged TT was noted in three patients: two at stage II and one at the beginning of stage III. A significant change was seen in ELT. The mean ELT, $215 \pm 77$ $\mathrm{min}$ at the time of induction of anesthesia, decreased progressively during the first stage of surgery. It reached a minimum value at the time of reperfusion of the homograft $(68 \pm 53 \mathrm{~min})$ and had returned toward the preoperative value at $2 \mathrm{hr}$ in stage III (III $+120 \mathrm{~min}$ ).

Intraoperative change in fibrinolytic activity in all patients is shown in Table 3 . Four patients had an ELT of less than $1 \mathrm{hr}$ at the beginning of surgery; that number increased to 21 by the time of homograft reperfusion. ELT improved toward preoperative values at the end of surgery, remaining shorter than $1 \mathrm{hr}$ in only one patient. A significant number of patients (15) had a slightly positive test for FDP at the beginning of surgery. The number increased to 26 patients by 2 $\mathrm{hr}$ after reperfusion of the homograft, and FDP remained positive in 22 patients at the conclusion of surgery, although the concentrations of FDP were never high.

\section{Thrombelastographic Variables}

The intraoperative change in TEG variables of patients with primary biliary cirrhosis is shown in Table 2 . Preoperative values indicated a slightly prolonged reaction time $(R)$. The maximum amplitude $(M A)$ and clot formation rate $(\alpha)$ were $54.6 \pm 11.2 \mathrm{~mm}$ and 55.5 $\pm 8.5^{\circ}$, respectively. The whole blood clot lysis index $\left(A_{60} / M A \times 100\right)$ was $83 \%$, which indicates whole blood clot lysis activity. The $R$ value was prolonged during the early phase of the third stage of surgery. It was prolonged significantly at the time of reperfusion of the homograft $(11.2 \pm 5 \mathrm{~min})$ and returned toward its preoperative value at $2 \mathrm{hr}$ in stage III $(8.8 \pm 3.0$ $\min )$. The changes in $R+k$ were similar to those in $R$. The whole blood clot lysis index decreased progressively from the beginning of surgery, and the lowest value was observed in the middle of the anhepatic stage. It improved after reperfusion of the homograft and returned toward the preoperative value at $2 \mathrm{hr}$ in stage III. Intraoperative change of whole blood clot lysis activity in all patients is shown in Table 3. Blood from three patients lysed within $2 \mathrm{hr}$ at induction of anesthesia; whole blood clot lysis was seen most frequently from the middle of stage II to $30 \mathrm{~min}$ into stage III. No patient's blood lysed within $2 \mathrm{hr}$ at the conclusion of surgery.

\section{Correlation of Thrombelastographic Variables and Coagulation Profile}

To determine the correlation between the conventional coagulation profile and the TEG variables, preoperative values were compared by linear regression analysis. In general, correlation between the variables of TEG and those of the coagulation profile was poor. 
Table 1. Preoperative Coagulation Profile of Liver Transplantation Patients

\begin{tabular}{lcccccccc}
\hline \multicolumn{1}{c}{ Variables } & Normal range & Neoplasm & $\begin{array}{c}\text { Primary biliary } \\
\text { cirrhosis }\end{array}$ & $\begin{array}{c}\text { Postnecrotic } \\
\text { cirrhosis }\end{array}$ & $\begin{array}{c}\text { Sclerosing } \\
\text { cholangitis }\end{array}$ & $\begin{array}{c}\text { Miscellaneous } \\
\text { Disease }\end{array}$ & Retransplant & All patients \\
\hline Number of patients & & 9 & 13 & 11 & 6 & 9 & 15 & 15 \\
PT (sec) & $10.8-13.0$ & $13.3 \pm 4.0$ & $13.9 \pm 1.7$ & $15.7 \pm 1.4$ & $13.3 \pm 3.6$ & $18.9 \pm 5.4$ & $13.2 \pm 1.5$ & $14.8 \pm 3.7$ \\
aPTT (sec) & $26-34$ & $34.1 \pm 6.3$ & $41.0 \pm 8.7$ & $40.2 \pm 4.1$ & $42.4 \pm 10.1$ & $46.7 \pm 10.6$ & $41.7 \pm 14.2$ & $41.5 \pm 13.9$ \\
Platelets $\left(1000 / \mathrm{mm}^{3}\right)$ & $150-450$ & $245 \pm 151$ & $181 \pm 199$ & $162 \pm 178$ & $86 \pm 19$ & $82 \pm 64$ & $116 \pm 115$ & $147 \pm 144$ \\
Fibrinogen $(\mathrm{mg} \mathrm{\% )}$ & $150-450$ & $323 \pm 166$ & $382 \pm 116$ & $247 \pm 134$ & $238 \pm 44$ & $167 \pm 86$ & $301 \pm 149$ & $292 \pm 138$ \\
Factor V (U/ml) & $0.5-1.5$ & $0.82 \pm 0.41$ & $0.54 \pm 0.27$ & $0.35 \pm 0.12$ & $0.47 \pm 0.20$ & $0.28 \pm 0.23$ & $0.66 \pm 0.29$ & $0.53 \pm 0.32$ \\
Factor VII (U/ml) & $0.5-1.5$ & $0.56 \pm 0.25$ & $0.83 \pm 0.35$ & $0.35 \pm 0.25$ & $0.71 \pm 0.49$ & $0.28 \pm 0.24$ & $0.53 \pm 0.26$ & $0.57 \pm 0.39$ \\
Factor VIII (U/ml) & $0.5-1.5$ & $1.42 \pm 0.33$ & $2.03 \pm 0.73$ & $1.82 \pm 0.93$ & $2.53 \pm 1.30$ & $2.30 \pm 0.70$ & $1.92 \pm 1.35$ & $2.00 \pm 0.90$ \\
ELT (min) & $>300$ & $218 \pm 48$ & $215 \pm 77$ & $163 \pm 87$ & $218 \pm 67$ & $132 \pm 68$ & $247 \pm 86$ & $202 \pm 81$ \\
\hline
\end{tabular}

Sixty-three patients, values are mean \pm SD.

Table 2. Intraoperative Changes in Coagulation of Patients with Primary Biliary Cirrhosis

\begin{tabular}{|c|c|c|c|c|c|c|c|c|}
\hline & \multirow[b]{2}{*}{ Before operation } & \multirow{2}{*}{$\frac{\text { Stage I }}{120 \mathrm{~min}}$} & \multicolumn{2}{|c|}{ Stage II } & \multicolumn{3}{|c|}{ Stage III } & \multirow[b]{2}{*}{ End } \\
\hline & & & $5 \mathrm{~min}$ & $30 \mathrm{~min}$ & $5 \mathrm{~min}$ & $30 \mathrm{~min}$ & $120 \mathrm{~min}$ & \\
\hline PT (sec) & $13.9 \pm 1.7$ & $13.7 \pm 1.0$ & $14.0 \pm 1.3$ & $13.8 \pm 1.2$ & $15.4 \pm 1.6^{a}$ & $15.7 \pm 1.5^{a}$ & $15.0 \pm 1.4$ & $14.6 \pm 1.4$ \\
\hline aPTT (sec) & $41.0 \pm 8.7$ & $40.2 \pm 7.7$ & $46.0 \pm 12.9$ & $51.2 \pm 16.0$ & $75.2 \pm 39.2^{\prime \prime}$ & $61.5 \pm 23.6^{a}$ & $46.0 \pm 9.7$ & $38.4 \pm 3.8$ \\
\hline Platelets $\left(1000 / \mathrm{mm}^{3}\right)$ & $181 \pm 199$ & $150 \pm 122$ & $149 \pm 120$ & $154 \pm 122$ & $124 \pm 63^{a}$ & $132 \pm 61$ & $134 \pm 82$ & $143 \pm 74$ \\
\hline Fibrinogen (mg \%) & $323 \pm 166$ & $288 \pm 100$ & $252 \pm 113$ & $235 \pm 88$ & $194 \pm 93^{a}$ & $195 \pm 101^{a}$ & $191 \pm 90^{a}$ & $210 \pm 92$ \\
\hline Factor $\mathrm{V}(\mathrm{U} / \mathrm{ml})$ & $0.54 \pm 0.27$ & $0.43 \pm 0.22$ & $0.35 \pm 0.19$ & $0.38 \pm 0.19$ & $0.26 \pm 0.18^{a}$ & $0.22 \pm 0.13^{a}$ & $0.21 \pm 0.11^{a}$ & $0.23 \pm 0.13^{a}$ \\
\hline Factor VII $(\mathrm{U} / \mathrm{ml})$ & $0.83 \pm 0.35$ & $0.71 \pm 0.37$ & $0.62 \pm 0.31$ & $0.65 \pm 0.27$ & $0.52 \pm 0.23^{a}$ & $0.52 \pm 0.18^{a}$ & $0.53 \pm 0.18$ & $0.51 \pm 0.17$ \\
\hline Factor VIII (U/ml) & $2.03 \pm 0.73$ & $1.81 \pm 0.86$ & $1.51 \pm 0.88$ & $1.49 \pm 0.75$ & $1.11 \pm 0.70^{a}$ & $0.82 \pm 0.53^{a}$ & $0.87 \pm 0.44^{a}$ & $0.93 \pm 0.35^{a}$ \\
\hline ELT (min) & $215 \pm 77$ & $134 \pm 93$ & $140 \pm 88$ & $101 \pm 77^{a}$ & $68 \pm 53^{a}$ & $108 \pm 45$ & $155 \pm 63$ & $185 \pm 70$ \\
\hline$R(\min )$ & $8.5 \pm 2.4$ & $8.2 \pm 3.6$ & $6.6 \pm 3.4$ & $7.5 \pm 2.3$ & $11.2 \pm 5.0^{a}$ & $9.7 \pm 2.7$ & $8.8 \pm 3.0$ & $8.0 \pm 2.9$ \\
\hline$R+k(\min )$ & $12.0 \pm 2.7$ & $12.0 \pm 6.3$ & $9.5 \pm 2.5$ & $11.0 \pm 2.2$ & $23.5 \pm 16.4^{a}$ & $15.0 \pm 6.1$ & $12.5 \pm 4.5$ & $10.0 \pm 2.6$ \\
\hline$\alpha\left(^{\circ}\right)$ & $55.5 \pm 8.5$ & $53.8 \pm 12.2$ & $53.6 \pm 10.4$ & $53.1 \pm 7.4$ & $39.1 \pm 17.5^{a}$ & $48.6 \pm 10.5$ & $50.6 \pm 12.0$ & $55.1 \pm 8.0$ \\
\hline$M A(\mathrm{~mm})$ & $54.6 \pm 11.2$ & $57.3 \pm 9.4$ & $52.3 \pm 11.0$ & $51.3 \pm 9.4$ & $38.2 \pm 14.5^{\prime \prime}$ & $51.7 \pm 8.9$ & $51.5 \pm 11.9$ & $52.1 \pm 10.8$ \\
\hline$A_{60} / M A \cdot 100(\%)$ & $83.0 \pm 7.8$ & $76.4 \pm 14.5$ & $73.9 \pm 27.0$ & $65.5 \pm 35.5$ & $75.8 \pm 34.4$ & $79.8 \pm 29.3$ & $92.2 \pm 8.6^{a}$ & $91.7 \pm 6.7^{a}$ \\
\hline
\end{tabular}

Eleven patients; values are mean \pm SD. "Significantly different from the corresponding preoperative value $(P<0.05)$.

However, the reaction time correlated best with aPTT $(r=0.49, P<0.001)$. MA correlated better with platelet count $(r=0.59, P<0.001)$ and fibrinogen level $(r=0.64, P<0.001)$. The whole blood clot lysis time $(F)$ did correlate with ELT $(r=0.54, P<0.05)$. There were 45 occasions when patients received platelets alone. The mean increase in platelet count was 40,200 $\pm 31,400 / \mathrm{mm}^{3}$ after infusion of 10 units of platelets; it correlated best with the $M A$ value, which increased $13.2 \mathrm{~mm}$. When 6 units of cryoprecipitate were given alone, factor I increased by $37 \mathrm{mg} \%$ and factor VIII by $13 \mathrm{U} / \mathrm{ml}$, while aPTT decreased by $5.7 \mathrm{sec}$. The corresponding changes in TEG variables were a decrease in reaction time by $1.2 \mathrm{~min}$ and an increase in clot formation rate by $9.4^{\circ}$.

\section{Comparison of Thrombelastograph-Monitored and Nonmonitored Groups}

The blood volume infused to patients in our series was compared with that required by a group of pa- tients who received liver transplants between March 1981 and January 1983, and who were not monitored by TEG. Five patients in the monitored group who received more than 100 units of $R B C$ transfusion were excluded from the analysis. Seventeen patients in the nonmonitored group were excluded from the comparison-five patients who required more than 100 units of RBC and 12 patients who underwent veno-venous bypass with systemic heparinization, some of whom developed uncontrollable bleeding. The preoperative diagnoses of patients in the two groups were not different $(P>0.5)$. The volume of blood given to patients in the two groups is shown in Table 4. Patients in the TEG-monitored group received $17 \pm 12.9$ units of RBC, whereas patients in the nonmonitored group required $26.7 \pm 23.8$ units of RBC $(P<0.05)$. A significant reduction in FFP infusion (18.3 \pm 12.5 vs $26.7 \pm 24.1$ units $)$ and a decrease in total fluids infused $(20.2 \pm 11.2$ vs $31.4 \pm 19.2 \mathrm{~L}$ ) were noted in patients of the TEGmonitored group. On the other hand, significantly 
Table 3. Intraoperative Changes in Number of Patients with Fibrinolytic Activity

\begin{tabular}{|c|c|c|c|c|c|c|c|c|c|}
\hline & \multirow[b]{2}{*}{ Before operation } & \multicolumn{2}{|c|}{ Stage I } & \multicolumn{2}{|c|}{ Stage II } & \multicolumn{3}{|c|}{ Stage III } & \multirow[b]{2}{*}{ Enc } \\
\hline & & $30 \mathrm{~min}$ & $120 \mathrm{~min}$ & $5 \mathrm{~min}$ & $30 \mathrm{~min}$ & $5 \mathrm{~min}$ & $30 \mathrm{~min}$ & $120 \mathrm{~min}$ & \\
\hline \multicolumn{10}{|l|}{ ELT } \\
\hline$>1 \mathrm{hr}$ & 48 & 41 & 25 & 35 & 29 & 27 & 21 & 37 & 43 \\
\hline$<1 \mathrm{hr}$ & 4 & 10 & 15 & 14 & 20 & 21 & 13 & 1 & 1 \\
\hline \multicolumn{10}{|l|}{ FDP } \\
\hline Negative & 36 & 37 & 36 & 37 & 31 & 34 & 30 & 27 & 31 \\
\hline Positive & 15 & 16 & 16 & 17 & 21 & 20 & 22 & 26 & 22 \\
\hline \multicolumn{10}{|l|}{$F$} \\
\hline$>2 \mathrm{hr}$ & 55 & 53 & 51 & 52 & 49 & 49 & 46 & 55 & 58 \\
\hline$<2 \mathrm{hr}$ & 3 & 5 & 7 & 6 & 10 & 10 & 12 & 3 & 0 \\
\hline
\end{tabular}

more platelets ( $20.8 \pm 12.8$ vs $14.1 \pm 13.7$ units) and cryoprecipitate ( $17.2 \pm 8.5$ vs $10.2 \pm 4.5$ units) were infused to the TEG-monitored patients. The number of donors from whom each patient received blood was similar between the two groups $(67.9 \pm 43.9$ vs 71.4 $\pm 63.4)$.

\section{Discussion}

As might be expected of patients with end-stage liver disease, preoperative coagulation profiles of many subjects studied showed prolonged PT and aPTT and decreased levels of factors produced by the liver. Coagulopathy was worse preoperatively in patients with miscellaneous types of liver disease and with postnecrotic cirrhosis because these patients have more parenchymal hepatocellular damage, which disrupts the synthesis of the blood coagulation factors. Other patients, such as those with neoplasms, may have close to normal hepatic function. Supranormal levels of fibrinogen and factor VIII activity were observed in many of our patients. A similar high level of factor VIII was found previously (14) and may result from increased production related to a stress response, release from damaged liver cells, or impaired catabolism of factor VIII (15). Preoperative fibrinogen level was lower than normal in only $20 \%$ of the patients. However, moderate prolongation of both TT and reptilase time was common and suggests a possible dysfibrinogenemia. Abnormal fibrin polymerization is known to occur in liver disease (16) and is reported to be related to an excessive sialic acid content in the fibrinogen molecule $(17,18)$. The clinical significance of the acquired dysfibrinogenemia in patients with liver disease and its role in patients undergoing liver transplantation requires further investigation.

Throughout the surgical procedure, a fluid composed of RBC, FFP, and Plasma-lyte $A$ in a ratio of 300:200:250 ml was used for volume replacement. RBC was mixed with FFP to supplement coagulation factors. Plasma-lyte A was added to decrease the loss of RBC and possibly to improve the circulatory rheology. Plasma-lyte A was chosen because it does not contain calcium, which can initiate clotting in the rapid infusion system; it does not contain glucose, which may cause hyperglycemia during massive blood transfusion; and it is isoosmolar. The blood mixture had levels of hematocrit, $27 \pm 2$; hemoglobin, $9 \pm$ $1.2 \mathrm{mg} \%$; factor I, $130 \mathrm{mg} \%$; factor II, $0.59 \mathrm{U} / \mathrm{ml}$; factor $\mathrm{V}, 0.21 \mathrm{U} / \mathrm{ml}$; factor VII, $0.58 \mathrm{U} / \mathrm{ml}$; and factor VIII, $0.57 \mathrm{U} / \mathrm{ml}$. Once blood volume was replaced by this mixture, platelets, fibrinogen, and factor VIII were replenished with transfusion of platelets or cryoprecipitate, guided by TEG monitoring. A coagulation factors-rich blood mixture was transfused, and the plasma levels of coagulation factors reached the levels in the transfused blood mixture rapidly as surgery progressed. The change is more likely due to a dilutional effect associated with surgical bleeding. As long as blood was simply entering the patient by transfusion and leaving by exsanguination, the dilutional effects should not bring the coagulation factors below those in the infused blood mixture. If fibrinolysis or intravascular coagulation occurred, differential falls in certain factors might be seen.

No attempt was made to correct coagulopathy during unheparinized veno-venous bypass in the anhepatic stage, in order to minimize possible thrombosis or embolization. Only RBC and FFP were infused to replace the blood volume. The increase in fibrinolytic activity was the most significant change in the coagulation pattern during the anhepatic stage. This relative lack of change in coagulation profile measurements may be due to the relatively short duration of the second stage $(89 \pm 21 \mathrm{~min}$; compared with 221 $\pm 80 \mathrm{~min}$, stage I; and $343 \pm 123 \mathrm{~min}$, stage III) or to the use of veno-venous bypass (19). It appears that the veno-venous bypass system, which used a heparin-coated Gott Aneurysm Shunt (Sherwood Medical, St. Louis, MO), did not interfere with the 
Table 4. Comparison of Transfused Blood Volume between Thrombelastograph-Monitored Patients and Nonmonitored Patients

\begin{tabular}{lcc}
\hline \multicolumn{1}{c}{ Blood products } & $\begin{array}{c}\text { TEG-monitored } \\
\text { patients } \\
(n=58)\end{array}$ & $\begin{array}{c}\text { Nonmonitored } \\
\text { patients } \\
(n=47)\end{array}$ \\
\hline Red blood cells (unit) & $17.0 \pm 12.9^{a}$ & $26.7 \pm 23.8$ \\
Fresh frozen plasma (unit) & $18.3 \pm 12.5^{\prime \prime}$ & $26.7 \pm 24.1$ \\
Platelets (unit) & $20.8 \pm 12.8^{a}$ & $14.1 \pm 13.7$ \\
Cryoprecipitate (unit) & $12.2 \pm 14.2^{\prime \prime}$ & $3.9 \pm 12.9$ \\
Total blood products (unit) & $67.9 \pm 43.9$ & $71.4 \pm 63.4$ \\
Crystalloid (litre) & $10.2 \pm 4.5^{a}$ & $17.2 \pm 8.5$ \\
Total volume infused (litre) & $20.2 \pm 11.2^{a}$ & $31.4 \pm 19.2$ \\
\hline
\end{tabular}

Values are mean \pm SD. "Significantly different from the corresponding values of the nonmonitored patients $(P<0.05)$.

coagulation system, by thrombosis or by coagulopathy, and may even have played a beneficial role. By decompressing the portal venous system and maintaining venous return from the lower extremities, it could have decreased venous pressure to decrease bleeding and reduced the congestive ischemia of the viscera, which in turn might have minimized the metabolic acidosis that could have adversely affected the blood coagulation system.

Reperfusion of the homograft was associated with significant coagulopathy, which was clearly demonstrated by the TEG measurements: prolonged $R$, prolonged $R+k$, decreased $M A$, decreased $\alpha$, and decreased $F$ (which was shortened to $92 \mathrm{~min}$, reflecting the decreased function of coagulation factors, including fibrinogen and platelets, and active whole blood lysis). These findings, in agreement with reports by Groth (6) and by von Kaulla et al. (4), might have resulted from pathophysiologic changes that occur on reperfusion of the graft liver. First, there is an exaggerated dilutional effect. The influx of the organ preservation solution from the donor liver may contribute further to the dilution of coagulation factors. Second, an active fibrinolytic process is evident in many patients. This could be due to release of tissue plasminogen activator from vascular endothelium (20) or to activation of protein C (21). Third, other investigators stressed disseminated intravascular coagulation (DIC) as a major cause of the change $(2,5)$, but there is little evidence of DIC in this study. Groth et al. suggested that loss of hepatic clearance function played a role in fibrinolytic activation or that antigen-antibody reaction might lead to DIC (2). However, the lack of high levels of circulating fibrin monomers and FDP found in this study does not support a major role for DIC as a cause of excessive bleeding. In addition, an attempt to identify microthrombi in major organs of a canine liver transplant recipient after clinical evidence of intravascular coagulation was not successful (22). No clinical sign of ischemic injury caused by microthrombi in major vital organs was seen in our patients. Fourth, the possibility of trapping of coagulation factors and platelets, especially by the poorly preserved donor liver, can be considered. However, the mean increase in platelet count after platelet infusion during the early part of the third stage of surgery was not different from that in the first stage. Fifth, as postulated by Stremple et al., levels of heparin or a heparin-like substance may be increased (23). However, of 13 patients who received the TT and reptilase time test, TT was prolonged significantly in only two patients, indicating that heparin or a heparin-like substance plays a rather minor role in coagulopathy. Finally, the effects of other humoral and metabolic factors should be emphasized. Metabolic acidosis, decrease in cardiovascular performance, decreased plasma ionized calcium level, decrease in body temperature, and hyperkalemia were observed during reperfusion in most patients; and all of these could adversely affect the coagulation system. The blood coagulability measured by TEG improved without significant changes in levels of coagulation factors $30 \mathrm{~min}$ after reperfusion in a significant number of patients. That is not sufficient time to expect the functional recovery of the donor liver, although it coincided with the recovery of the above physiologic variables. Thus metabolic and humoral changes that occur with the reperfusion of the homograft may interfere with the hemostatic process more than has been recognized.

All coagulation profile and TEG variables started to improve $30 \mathrm{~min}$ after reperfusion of the liver and, with the exception of factor V and factor VIII, all the factor levels and ELT returned toward the baseline value within $2 \mathrm{hr}$ after reperfusion. This agrees with the findings of Groth (6), and it appears that recovery of the coagulation system coincides with bile production and the metabolism of citrate (Marquez JM, unpublished data, 1984).

In this series of patients, blood replacement therapy was guided by TEG monitoring. TEG, developed in the 1950s, has been used mostly for research purposes and is a simple, reliable, and sensitive method. A general assessment of blood coagulation status can be obtained within $30 \mathrm{~min}(8,24)$, and the coagulation process is assessed continuously and rapidly in the operating room. TEG measures the coagulation process in whole blood, rather than testing blood coagulability in anticoagulated plasma, as is commonly done to obtain the conventional analytic coagulation profile. The interpretation of TEG and its relation to the conventional coagulation profile has been estab- 
lished by several researchers $(25,26)$. The reaction time $(R)$ is closely related to aPTT. The maximum amplitude $(M A)$ is a reflection of platelet function and activity of factor XIII. The rate of clot formation $(\alpha)$ primarily reflects the function of fibrinogen, and it increases with improved platelet function. The whole blood clot lysis time $(F)$ is reported to be closely related to ELT. To express the activity of whole blood clot lysis, the ratio $A_{60}: M A$ was used.

In general, the correlation between the TEG variables and coagulation profile measurements in the patients studied is similar to results published by others, although it was not high in these patients, probably because of both the large standard deviations in the individual data and other physiologic changes. Since replacement of blood products was guided by the TEG monitoring, as described in Methods, there were fewer significant changes in $R, R+k, M A$, and $\alpha$ values during the first stage, although there were decreases in coagulation factors and platelets. This discrepancy may indicate that blood coagulability was maintained in spite of low but higher than critical levels of coagulation factors.

The effectiveness of the TEG-guided replacement therapy is shown in a representative TEG pattern (Fig. 4). Poor preoperative coagulation status was improved by selective transfusion of FFP, platelets, and cryoprecipitate; and blood coagulability was normal at the end of surgery after transfusion of 46 units of RBC, 48 units of FFP, 20 units of platelets, and 12 units of cryoprecipitate. The clinical significance of TEG-guided replacement therapy is demonstrated in the volumes patients received during the surgical procedure. Treatment of patients in the control group, who received liver transplants from 1981 to 1982, was guided by clinical impression and the results of PT, aPTT, and platelet count. Since TEG monitoring began, the required volume of fluid infusion has decreased by $33 \%$. Defects in the coagulation system were treated with platelets or cryoprecipitate when needed. All but two patients in the TEG-monitored group received veno-venous bypass, and the improved surgical technique could have contributed to the reduction in blood loss. However, because of the magnitude of the intraoperative bleeding, a controlled, randomized study could not be justified. Furthermore, it is not unreasonable to speculate that patients in the TEG-monitored group had better blood coagulability because TEG patterns were normal toward the end of surgery. The total number of blood donors to which patients were exposed remained similar, even though patients in the TEG-monitored group received more platelets and cryoprecipitate; therefore exposure to hepatitis or acquired immune deficiency

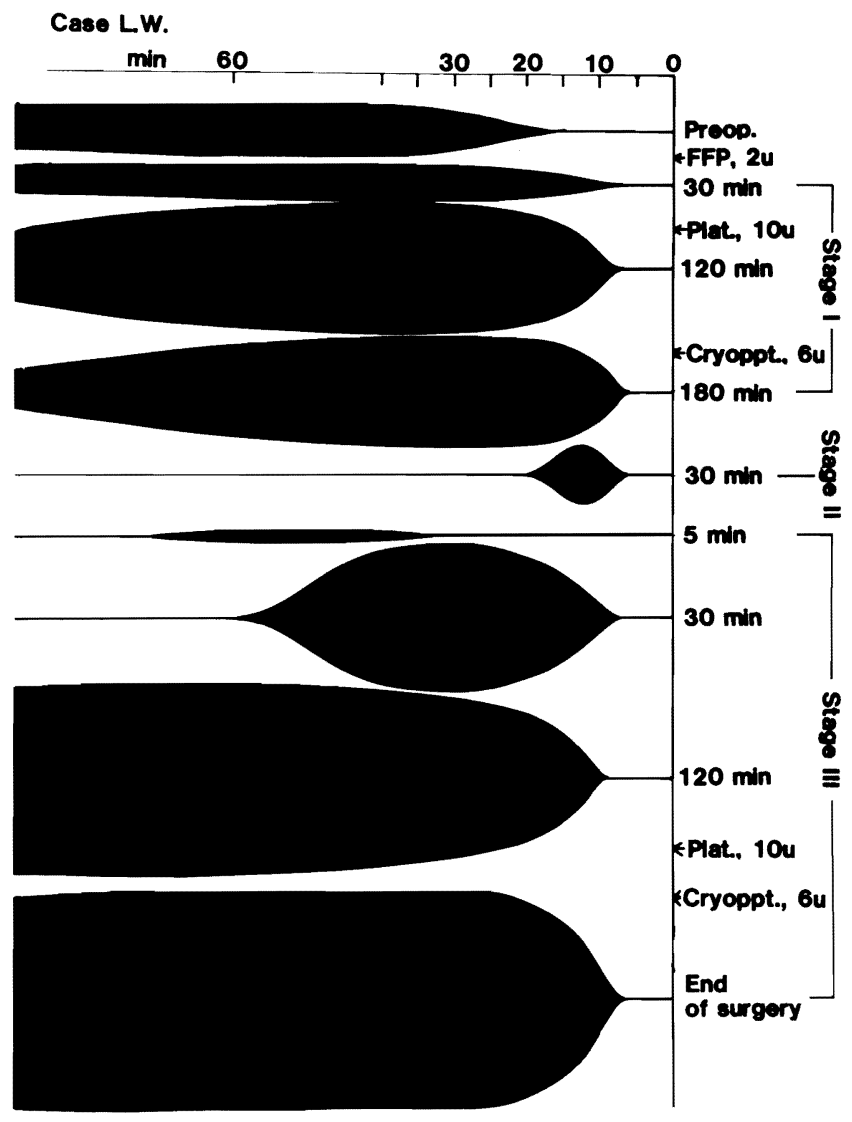

Figure 4. Thrombelastographic pattern of one patient. Reaction time, maximum amplitude, and clot formation rate improved after sequential transfusion of FFP, platelets, and cryoprecipitate. Rapid whole blood clot lysis was demonstrated during the second stage and the early part of the third stage of surgery. Transfusion of platelets and cryoprecipitate normalized the TEG pattern at the end of the operation. This patient was transfused 46 units of RBC, 48 units of FFP, 20 units of platelets, and 12 units of cryoprecipitate.

syndrome was not increased in patients of the TEGmonitored group.

In this series of 66 patients, very severe coagulopathy was observed ranging from no clotting at all to a rapid whole blood clot lysis (within $0.5 \mathrm{hr}$ ), which often was associated with blood transfusions in excess of $50 \mathrm{~L}$. Normalizing the levels of platelets and coagulation factors may not always be possible. However, maintaining the blood coagulability is critically important for these patients who have marginal or no liver function. This study showed intraoperative changes in coagulation and the clinical effectiveness of TEG monitoring and TEG-guided replacement therapy. Further investigation is required for a better understanding of the clinical use of TEG and antifibrinolytic therapy to reduce morbidity and mortality in patients undergoing liver transplantation. 
The authors thank Mr. Jeffrey M. Yoffe and Mr. Mark Zapp for their technical assistance, Ms. Lisa Cohn for her editorial help, Dr. Ray McKenzie for his support, and the Hellige Company of Germany for the loan of equipment.

\section{References}

1. Starzl TE, Iwatsuki S, Van Thiel DH, et al. Evolution of liver transplantation. Hepatology 1982;2:614-36.

2. Groth CG, Pechet L, Starzl TE. Coagulation during and after orthotopic transplantation of the human liver. Arch Surg 1969;98:31-4.

3. Toghill PJ, Green S, Ferguson R. Platelet dynamics in chronic liver disease with special reference to the role of spleen. J Clin Pathol 1977;30:367-71.

4. von Kaulla KN, Kaye H, von Kaulla E, Marchioro TL, Starzl TE. Changes in blood coagulation, before and after hepatectomy or transplantation in dogs and man. Arch Surg 1966;92:71-9.

5. Flute PT, Rake MO, Williams R, Seaman MI, Calne RY. Liver transplantation in man-IV, haemorrhage and thrombosis. $\mathrm{Br}$ Med J 1969;3:20-3.

6. Groth CG. Changes in coagulation. In: Starzl TE, ed. Experience in hepatic transplantation. Philadelphia: WB Saunders, 1969;159-75.

7. Pechet L, Groth CG, Daloze PM. Changes in coagulation and fibrinolysis after orthotopic canine liver transplantation. J Lab Clin Med 1969;73:91-102.

8. Howland WS, Castro EB, Fortner JB, Gould P. Hypercoagulability-thrombelastographic monitoring during extensive hepatic surgery. Arch Surg 1974;108:605-8.

9. Lewis JH. Coagulation defects. JAMA 1961;178:1014.

10. Lewis JH. Hemostasis and hemorrhage. Sci Clin 1971;1:1-66.

11. Lewis JH, Spero JA, Hasiba U. Diagnostic methods: laboratory tests. In: Bleeding disorders. Garden City, NY: Medical Exam Publishing Co, Inc, 1978:22-34.

12. De Nicola P. Thrombelastography. Springfield, IL: Charles C Thomas, 1957.

13. Raviv G, Cramon DB, Epstein M, Zuckerman L, Caprini JA.
Electronic readout for three-channel thrombelastograph. J Med 1978;9:17-31.

14. Bontempo FA, Lewis $\mathrm{JH}$, Van Thiel $\mathrm{DH}$, et al. The relation of preoperative coagulation findings to diagnosis, blood usage and survival in adult liver transplantation. Transplantation (in press).

15. Green AJ, Ratnoff OD. Elevated antihemophilic factor (AHF, factor VIII) procoagulant activity and AHF-like antigen in alcoholic cirrhosis of the liver. J Lab Clin Med 1974;83:189-97.

16. Ragni MV, Lewis JH, Spero JA, et al. Bleeding and coagulation abnormalities in alcoholic cirrhotic liver disease. Clin Exp Res 1982;6:267-74.

17. Fletcher AP, Biederman O, Moore D, Alkjaersig N, Sherry S. Abnormal plasminogen-plasmin system activity (fibrinolysis) in patients with hepatic cirrhosis: its cause and consequences. J Clin Invest 1964;43:681-95.

18. Martinez J, Palascak JE, Kwasniak D. Abnormal sialic acid content of the dysfibrinogenemia associated with liver disease. J Clin Invest 1978;61:535-8.

19. Shaw BW, Martin MJ, Marquez JM, et al. Venous bypass in clinical liver transplantation. Ann Surg 1984;200:524-34.

20. Collen D. On the regulation and control of fibrinolysis. Thromb Haemostas 1980;43:77.

21. Esmon C, Owen W. Identification of an endothelial cell cofactor for thrombin-catalyzed activation of protein C. Proc Natl Acad Sci USA 1981;78:2249.

22. Hutchinson DE, Genton E, Porter KA, et al. Platelet changes following clinical and experimental hepatic homotransplantation. Arch Surg 1968;97:27-33.

23. Stremple JF, Hussey CV, Ellison EH. Study of clotting factors in liver homotransplantation. Am J Surg 1966;111:862-9.

24. Kang YG, Abouleish E. Thrombelastography in obstetrics (Abstract). Anesthesiology 1981;55(suppl):A304.

25. Zuckerman L, Cohen E, Vagher JP, Woodward E, Caprini JA. Comparison of thrombelastography with common coagulation tests. Thromb Haemostas 1981;46:752-6.

26. Howland WS, Schwizer O, Gould P. A comparison of intraoperative measurements of coagulation. Anesth Analg 1974;53:657-63. 